\title{
Study on C-reactive Protein in Periodontal Diseases: Estimation and Correlation in Health, Gingivitis and Periodontitis and its Assessment after Scaling and Root Planing
}

Rajiv Saini

\begin{abstract}
Background: Periodontitis is associated with elevated levels of systemic inflammatory biomarker C-reactive protein (CRP). This study is outlined to determine and correlate the C-reactive CRP level in health, gingivitis and periodontitis and to assess the CRP values after complete scaling and root planing.
\end{abstract}

Materials and methods: A total of 60 systemically healthy subjects enrolled in the study. Out of which 20 subjects each categorized under three groups, i.e. periodontally healthy, gingivitis and periodontitis. Clinical parameters and serum CRP is recorded at the baseline, 15th and 30th day.

Results: Patients with chronic periodontitis had statistically significant elevations in serum CRP levels as compared to gingivitis and absence of detection of serum CRP level in health group. Results showed a highly significant change in clinical parameters and serum CRP with regard to improvement in the gingival inflammation and reduced bleeding on probing and plaque accumulation from the baseline when compared to the 15 th day and 30th day.

Conclusion: A correlation exists between the periodontal disease and CRP, as the periodontal disease regresses the value of CRP lowers down significantly. Nonsurgical periodontal treatment, i.e. scaling and root planing was effective in reducing the levels of serum CRP.

Keywords: CRP, Periodontitis, Inflammation, Biomarker.

How to cite this article: Saini R. Study on C-reactive Protein in Periodontal Diseases: Estimation and Correlation in Health, Gingivitis and Periodontitis and its Assessment after Scaling and Root Planing. Int J Experiment Dent Sci 2014;3(1):4-7.

Source of support: Nil

Conflict of interest: None declared

\section{INTRODUCTION}

The oral cavity has the potential to harbor at least 600 different bacterial species, and in any given patient, more than

\section{Associate Professor}

Department of Periodontology and Oral Implantology Rural Dental College, Pravara Institute of Medical Scinces Ahmednagar, Maharashtra-413736, India

Corresponding Author: Rajiv Saini, Associate Professor Department of Periodontology and Oral Implantology Rural Dental College, Pravara Institute of Medical Sciences, Loni Rahata, Ahmednagar, Maharashtra-413736, India, e-mail: drperiodontist@yahoo.co.in
150 species may be present, surfaces of teeth can have as much as billion bacteria in its attached bacterial plaque. ${ }^{1}$ Periodontitis has been proposed as having an etiological or modulating role in cardiovascular, cerebrovascular disease, diabetes, respiratory disease and adverse pregnancy outcome; several mechanisms have been proposed to explain or support such theories. Microorganisms in dental plaque interact with neutrophils/monocytes resulting in acute-phase inflammatory response by the systemic dissemination of locally produced mediators, such as $\mathrm{C}$-reactive protein (CRP), interleukins-1 beta (IL-1 $\beta$ ), interleukins-6 (IL-6) and tumor necrosis factor alpha (TNF- $\alpha){ }^{2}$ Thus, the acute-phase response might be useful as biomarkers of periodontitis contribution to systemic disease, as well as providing a prospective mechanistic link between the local and systemic manifestations of periodontitis. So, this study was outlined to determine and correlate the CRP level in health, gingivitis and periodontitis and to assess the CRP values after complete scaling and root planing.

\section{MATERIALS AND METHODS}

The present study was conducted in the department of periodontology, Rural Dental College, Loni in co-ordination with department of microbiology, Rural Medical College and central collection laboratory of Pravara Institute of Medical Sciences, Loni. The research protocol was approved by the University Research and Ethical Committee.

\section{Study Population}

The subjects enrolled in this study were selected from the out patient department of periodontology, Rural Dental College and Hospital, Loni. After an informed consent, a total 60 patients between the age of 18 and 65 years enrolled in the study as illustrated in Table 1. Exclusion criteria included: (1) presence of any systemic neurological disorder (e.g. epilepsy or schizophrenia), (2) presence of a disease with possible effects on the immune system (e.g. chronic infections or cancer), (3) patient, who have received antibiotics or non-steroidal anti-inflammatory drugs (NSAIDs) (like Ibuprofen) in past 9 to 11 weeks, (4) patients who have 
Table 1: Distribution of patients in study groups

\begin{tabular}{lll}
\hline Groups & Patient criteria & No. of patients \\
\hline A & Healthy patients & 20 \\
B & Gingivitis patients & 20 \\
C & Periodontitis patients & 20 \\
\hline
\end{tabular}

received periodontal treatment in past 6 months, (5) pregnant and lactating mother, (6) patient with artificial prosthesis, (7) patients who smoke or consume tobacco in any form, (8) patients suffering with diabetes, arthritis, any type of heart disease myocardial infarction and chronic heart disease (MI, CHD, etc.), (9) female patients using intrauterine birth control devices or birth control pills, (10) obese individuals (30 and above range as per WHO BMI cutoff for weight categories for Asians), (11) participants not willing to participate in the study.

\section{Clinical Protocol}

Clinical parameters of periodontal disease evaluated in this study were gingival index (Loe and Silness Index), plaque index (Turesky-Gilmore-Glickman modification of Quigley Hein Plaque Index), probing depth (PD) and clinical attachment level (CAL).

Baseline clinical and biochemical measurements were recorded of all the three groups. Full mouth scaling and root planing was carried out in patients belonging to groups $B$ and C. Recall visits is scheduled for all the subjects belonging to groups $\mathrm{B}$ and $\mathrm{C}$ on 15 th and 30th day, both clinical and biochemical measurements were recorded. Venous blood samples were obtained from the antecubital vein by venipuncture using $2 \mathrm{ml}$ syringe from each subject from all the three groups at baseline. On 15th and 30th day after scaling and root planing post-baseline blood samples obtained from only groups B and C. Samples were then shifted to laboratory and processed with centrifuge machine at rate of 10,000 rpm to separate the serum and plasma from the blood. Serum was then analyzed for CRP using a commercially available kit.

\section{C-reactive Protein (CRP) Test}

RHELAX CRP slide test kit was used for the in vitro detection of CRP in human serum by qualitative and quantitative rapid latex slide test. RHELAX CRP slide test for detection of CRP is based on the principle of agglutination. The test specimen (serum) is mixed with RHELAX CRP latex reagent and allowed to react. If CRP concentration is greater than $0.6 \mathrm{mg} / \mathrm{dl}$ a visible agglutination is observed. If CRP concentration is less than $0.6 \mathrm{mg} / \mathrm{dl}$, then no agglutination is observed. No special preparation of the patient was required prior to specimen collection. Two milliliter venous blood was collected into sterile disposable tube and processed with centrifuge at 10,000 rpm for serum separation. Only serum was used for testing. If there was delay in testing occurs then samples were stored at 2 to $8^{\circ} \mathrm{C}$.

\section{RESULTS AND DISCUSSION}

The gingival index and plaque index of experimental period, i.e. on baseline, 15 th and 30th day were recorded and compared as illustrated in Table 2. Results showed a highly significant change with regard to improvement in the gingival inflammation and reduced bleeding on probing and plaque accumulation from the baseline when compared to the 15 th and 30th day. In this study, the mean gingival index in all the three groups, i.e. health group (control group), gingivitis group and periodontitis group at baseline were $0.906 \pm 0.133,2.176 \pm 0.114$ and $2.6069 \pm 0.265$, respectively. Comparison of mean value of gingival scores at baseline between gingivitis group and control group, periodontitis group and control group, gingivitis group and periodontitis group was highly significant $(\mathrm{p}<0.01$, ' $t$ '-value $=33.42,26.15,6.82$ ). The mean gingival indices in gingivitis group on 15 th and 30 th day was $1.03 \pm 0.185$ and $0.75 \pm 0.009$, the mean gingival index (GI) in periodontitis group at 15 th and 30 th day is $1.55 \pm 0.252$ and $0.78 \pm 0.083$. Comparison of mean value of gingival scores at 15 th day between gingivitis group and periodontitis group was highly significant $(\mathrm{p}<0.01$, 't'-value $=7.56)$, while at 30 th day was not significant $(p>0.05$, ' $t$ '-value $=7.56)$. There was highly significant difference between mean values of GI within gingivitis group and periodontitis group at baseline to 15 th day and baseline to 30 th and 15 th to 30 th days (i.e. $p<0.01$ ). This study revealed that mean plaque index in all the three groups, i.e. health group, gingivitis group and periodontitis group at baseline were $0.982 \pm 0.079,2.17 \pm 0.12$ and $2.54 \pm 0.26$ respectively. Comparison of mean value of plaque scores at baseline between gingivitis group and control group, periodontitis group and control group, gingivitis group and periodontitis group were highly significant $(\mathrm{p}<0.01$, ' $t$ '-value $=36.96,25.66,5.78$ ). The mean plaque index in gingivitis group at 15 th and 30 th day was $1.52 \pm 0.13$ and $1.064 \pm 0.09$; the mean plaque index in periodontitis group at 15 th and 30th day was $2.02 \pm 0.012$ and $1.12 \pm 0.11$. Comparison of mean value of plaque scores at 15 th day between gingivitis group and periodontitis group was highly significant $(\mathrm{p}<0.01$, ' $\mathrm{t}$ '-value $=12.28)$, while at 30 th day was not significant $(\mathrm{p}>0.05$, 't'-value $=1.82)$. There was highly significant difference between mean values of PI within gingivitis group and periodontitis group at baseline to 15 th day and baseline to 30 th and 15 th to 30 th days (i.e. $\mathrm{p}<0.01)$.

Gains in (CAL) and reduction in pocket probing depth are the most common parameters used to measure clinical improvement. There was a significant decrease in the CAL 
Table 2: Clinical parameters and C-reactive protein levels of all the groups under study

\begin{tabular}{lllll}
\hline Groups & Variable & Mean & $\begin{array}{l}\text { Standard } \\
\text { deviation }\end{array}$ & $\begin{array}{l}\text { Standard } \\
\text { error (SE) }\end{array}$ \\
\hline A & GI & 0.91 & 0.13 & 0.03 \\
(Healthy) & PI & 0.98 & 0.08 & 0.018 \\
B & CRP & 0 & 0 & 0.024 \\
(Gingivitis) & GI BL & 2.18 & 0.11 & 0.040 \\
& GI 15 D & 1.03 & 0.18 & 0.020 \\
& GI 30 D & 0.75 & 0.09 & 0.027 \\
PI BL & 2.17 & 0.12 & 0.029 \\
PI 15 D & 1.52 & 0.13 & 0.022 \\
PI 30 D & 1.06 & 0.099 & 0.15 \\
C & CRP BL & 1.65 & 0.68 & 0.08 \\
CRP 15 D & 0.24 & 0.35 & 0 \\
& CRP 30 D & 0 & 0 & 0.06 \\
CGI BL & 2.61 & 0.26 & 0.056 \\
GI 15 D & 1.55 & 0.25 & 0.018 \\
GI 30 D & 0.78 & 0.083 & 0.058 \\
PI BL & 2.54 & 0.26 & 0.027 \\
PI 15 D & 2.02 & 0.12 & 0.024
\end{tabular}

BL: Base line; 15D: 15th day; 30D: 30th day; GI: Gingival index; PI: Plaque index; CRP: C-reactive protein

between baseline and 30th day. Statistically significant reduction in the probing pocket depth was also observed at the end of 30th day. The CAL in periodontitis group at baseline to 15 th and 30th day were $6.06 \pm 0.78,4.88 \pm 0.91$ and $4.41 \pm 0.82$ respectively.

The primary thrust of this study was to evaluate and correlate serum CRP which is potential indicator of disease activity. Data from this study revealed that CRP value varied according to periodontal disease. Mean CRP value in all the three groups, i.e. health group, gingivitis group and periodontitis group at baseline were $0.0 \pm 0.0$, $1.65 \pm 0.68$ and $1.95 \pm 0.65$ respectively. Comparison of mean value of $\mathrm{CRP}$ at baseline between gingivitis group and health group (control), periodontitis group and health group were highly significant ( $\mathrm{p}<0.01$, ' $\mathrm{t}$ '-value $=10.86$ and 13.45) while in gingivitis group and periodontitis group at baseline was not significant ( $p>0.05$, ' $t$ '-value $=1.43$ ) . The mean CRP value in gingivitis group at 15 th and 30 th day was $0.24 \pm 0.35$ and $0.0 \pm 0.0$; the mean CRP value in periodontitis group at 15 th and 30th day was $0.51 \pm 0.43$ and $0.0 \pm 0.0$. Comparison of mean value of CRP scores at 15 th day between gingivitis group and periodontitis group was highly significant $(\mathrm{p}<0.01$, ' $\mathrm{t}$ '-value $=2.18$ ), while at 30th day it was not significant $(p>0.05$, ' $t$ '-value $=0)$. There was highly significant difference between mean values of CRP within gingivitis group and periodontitis group at baseline to 15 th day and baseline to 30th and 15th to 30th days (i.e. $\mathrm{p}<0.01$ ). The mean score in this study for CRP level at baseline was reduced at the end of 15th day and further reduced to $0.0 \pm 0.0$ at 30 th day and this improvement can be attributed to the fact that the CRP are produced in inflammatory conditions and once the inflammation subsided due to treatment the CRP level returned to normal. This study has demonstrated that the extent of increase in CRP levels in gingivitis and periodontitis patients depends on severity of disease and elevation of CRP was associated with the presence of dental plaque.

This study found a similar correlation with the previous studies where they found as positive correlation between CRP and periodontitis patients. ${ }^{3-5}$ In this study, we observed a significant improvement in gingival score and plaque score following SRP, as documented in previous study ${ }^{6}$ and also this study correlates with the previous study that found a significant decrease in serum CRP, but only after a period of 6 months. ${ }^{7}$ However, it should be noted that CRP levels of patients tended to be higher at baseline and declined at reassessment suggesting that destructive periodontal disease are treatable and it may be possible to lower CRP value through effective management of destructive periodontal disease.

Conclusively, it could be stated from this study that, there seems to be strong correlation between periodontal diseases and CRP level and as there is progression and remission of periodontal disease; $C R P$ values follows the same. This study also concluded that there is positive effect of nonsurgical periodontal therapy, i.e. scaling and root planing on lowering the serum CRP level. 
This study also pursues certain limitations that should be over powered when future studies are proposed in context with correlation of serum CRP and periodontal status. Further large scale and longitudinal studies are clearly needed to clarify the relationship between periodontal infections and systemic inflammatory markers like CRP. In this study, we have used a chair side diagnostic test kit for the estimation of CRP, which has a lower detection limit of $6 \mathrm{ug} / \mathrm{ml}$ which is equivalent to $6 \mathrm{mg} / \mathrm{l}$ so more specific and high sensitive CRP (hsCRP) test should be used to draw even the minor readings from the serum.

\section{REFERENCES}

1. Saini R, Saini S, Sharma S. Oral sex, oral health and urogenital infections. J Glob Infect Dis 2010;2(1):57-62.

2. Saini R, Saini S, Saini SR. Periodontal diseases: a risk factor to cardiovascular disease. Ann Card Anaesth 2010;13(2):159-161.
3. Slade GD, Offenbacher S, Beck JD, Heiss G, Pankow JS. Acutephase inflammatory response to periodontal disease in the US population. J Dent Res 2000;79(1):49-57.

4. Ide M, McPartlin D, Coward PY, Crook M, Lumb P, Wilson RF. Effect of treatment of chronic periodontitis on levels of serum markers of acute-phase inflammatory and vascular responses. J Clin Periodontol 2003;30(4):334-340.

5. Ebersole JL, Machen RL, Steffen MJ, Willmann DE. Systemic acute-phase reactants, C-reactive protein and haptoglobin, in adult periodontitis. Clin Exp Immunol 1997;107(2): 347-352.

6. Cugini MA, Haffajee AD, Smith C, Kent RL Jr, Socransky SS. The effect of sealing and root planing on the clinical and microbiological parameters of periodontal disease: 12 month results. J Clin Periodontol 2000;27(1):30-36.

7. D'Aiuto F, Parkar M, Andreou G, Suvan J, Brett PM, Ready D, Tonetti MS. Periodontitis and systemic inflammation: control of the local infection is associated with reduction in serum inflammatory markers. J Dent Res 2004;83(2):156-160. 